\title{
A Composition of Fuzzy Sets
}

\author{
Vitaly I. Levin
}

Mathematics Department, Penza State Technological University

1-a, Baidukov pr., Penza, 440039, Russia

e-mail: vilevin@mail.ru

\begin{abstract}
:
A new operation on fuzzy sets - the $r$-composition of $n$-sets - is introduced. The particular cases of this operation are logical conjunction $(r=1)$ or disjunction $(r=n)$. In the general case $(1<r<n)$, this operation is purely fuzzy and has no analogs among the operations on fuzzy sets. The operation of $r$-composition is applied to the solution of control problems under uncertainty.

Keywords: fussy sets, logical operations, composition, uncertainty, control problems
\end{abstract}

\section{Introduction}

When solving control problems, it is frequently necessary to obtain a quantitative estimate for a certain situation under uncertainty using the evaluations of this situation given by a certain number of independent experts under the same conditions. The presence of uncertainty factor results in evaluations given in the form of the corresponding fuzzy subsets of the set of all possible alternatives. Note that the problem of obtaining an objective quantitative estimate of the studied situation reduces to the integration of individual evaluations of particular experts according to a certain reasonable criterion. Usually, the operation of intersection of fuzzy sets corresponding to individual evaluations of particular experts is used as a base for the integration rule. The new fuzzy set obtained as a result of the intersection is taken as the desired cooperative evaluation of the situation under study [1].

The disadvantages of the cooperative evaluation obtained in this way are its narrowness and lack of reliability. The first disadvantage means that the evaluation set is usually considerably narrower (contains less elements) than the evaluations made by particular experts and may be empty, especially if number of experts is sufficiently large. The second means that the elements that belong to evaluation set usually have small grades of membership to it, especially if experts are sufficiently independent, which results in noticeable differences in their opinions. This approach may be refined in one way or another [2], [4]. However, this refinement does not change its nature. A possible way out that allows us to solve the problem suggests rejecting idea of [1] to choose common part (intersection) of all 
individual evaluations as a cooperative evaluation and to replace it with a more flexible and productive principle of choice. This principle takes as a cooperative evaluation the individual evaluation given by a specially constructed "most representative" expert. It is obvious that, at each point of the domain of all possible alternatives, this expert must choose, as a measure of membership of this point to cooperative evaluation, an evaluation among the ones proposed by different experts that, in the general case, is distant from the extremal evaluations produced in this collective and has some "middle" position. And this choice means that the integration of individual expert evaluations into a cooperative one is not made on the basis of operations of fuzzy sets intersection (where minimal estimate of membership is taken) or union (maximal estimate is taken). This does not mean that any other known operations on fuzzy sets are used either. Operation required is a new operation on such sets, namely, their ordered choice. The goal of this paper is to describe this operation and to apply it to problem of making cooperative decisions under uncertainty to solve control and many other problems.

\section{Problem Statement}

Assume that we deal with a collective of $n$ independent experts, which quantitatively evaluate the same situation under uncertainty conditions (incomplete information). Suppose that the evaluation given by an arbitrary $i$-th expert has the form of a fuzzy subset of the set of all possible alternatives and is characterized by the corresponding membership function $M_{B_{i}}(X)$. The problem is to integrate (aggregate) individual evaluations of particular experts into one, cooperative, evaluation of the considered situation. In other words, it is necessary to determine an integrated, cooperative, evaluation set $B$ from some individual evaluation sets $B_{i}, i=\overline{1, n}$. As was mentioned in the Sec. 1, the conventional approach to the integration of individual evaluations into a cooperative one uses the intersection of fuzzy sets $B_{i}$ to obtain a new fuzzy set

$$
B=\overbrace{i=1}^{n} B_{i}, \quad M_{B}(x)=\min _{1 \leq i \leq n} M_{B_{i}}(x),
$$

which is taken as a cooperative evaluation of the considered situation. However, in view of the disadvantages specified above, this approach is not advisable. Therefore, we suppose that the individual evaluations of particular experts $B_{i}, i=\overline{1, n}$, are integrated into a cooperative evaluation $B$ by constructing the most representative expert who performs the ordered choice from measures of membership $M_{B_{i}}(x), i=\overline{1, n}$ of any point (alternative) $x$ to individual evaluations $B_{i}$. As a result, we obtain the measure of membership $M_{B}(x)$ for the cooperative evaluation $B$ (see Sec. 1). Then, from the mathematical standpoint, the posed problem reduces to constructing and studying properties of

appropriate functions of ordered choice from membership functions $M_{B_{i}}(x), i=\overline{1, n}$, and operations on fuzzy sets (individual expert evaluations) $B_{i}, i=\overline{1, n}$, described by these functions that lead to the fuzzy set (cooperative evaluation) $B$.

\section{Mathematical Apparatus}

It is known that the application of continuous logic (CL) with the support $A=[0,1]$ and logical 
operations of $\vee=\max ($ disjunction), $\wedge=\min$ (conjunction), and negation $\bar{x}=1-x$ allows one to generalize the set-theoretic operations to the case of fuzzy sets [5], [6].

$$
M_{A \cup B}(x)=\max \left(M_{A}(x), M_{B}(x)\right), M_{A \cap B}(x)=\min \left(M_{A}(x), M_{B}(x)\right), M_{\bar{A}}(x)=1-M_{A}(x) .
$$

Here, $M_{B}(x)$ is the measure of membership of the element $x$ to the set $B$. It can be seen that the measure of membership of an element to the union (intersection) of two fuzzy sets is defined as disjunction (conjunction) of the continuous logic of measures of membership of this element to each particular set, while the measure of membership of this element to the complement of the fuzzy set is the negation of the measure of membership to this set. The operations of the union and intersection of several fuzzy sets are introduced similarly to (2).

Let us introduce family of new operations of the composition of fuzzy sets. First, we note that the operations of the union and intersection of fuzzy sets (2) are a generalization of the operations of the union and intersection of conventional sets to the case of fuzzy sets that uses well-known operations of fuzzy logic (FL), namely, conjunction and disjunction. The application of new operations that generalize FL-logical determinants (LD) provides a new family of operations on fuzzy sets that have no analogs in operations on conventional sets and reflect more completely the fuzzy nature of the boundaries of fuzzy sets. For this purpose, we introduce a finite set

$$
A=\left\{a_{1}, a_{2}, \ldots, a_{n}\right\}
$$

where the $r$-th element in magnitude is $a^{r}$, so that $a^{1} \leq a^{2} \leq \ldots \leq a^{n}$. The function

$$
A \rightarrow a^{r}, r=\overline{1, n}
$$

is called the ordinal logical determinant (LD) of the rank $r$ and is denoted by $A^{r}$ or $\left|a_{i}\right|^{r}=\left|a_{1}, \ldots, a_{n}\right|^{r}$. The LD $A^{r}$ is the numerical characteristic of the set $A$, which is similar to the determinant of a square matrix. It is expressed in terms of its elements by using operations of FL in form (3):

$$
A^{r}=\bigvee_{i_{1} \neq \ldots \neq i_{n-r+1}} a_{i_{1}} \wedge \ldots \wedge a_{i_{n-r+1}}
$$

Consider a finite collection of fuzzy sets

$$
B=\left\{B_{1}, B_{2}, \ldots, B_{n}\right\}
$$

Let us introduce a family of operations on this collection

$$
B_{1}(r) B_{2}(r) \ldots(r) B_{n}=\underset{i=1}{(r)} B_{i}, \quad r=\overline{1, n}
$$

determined by following relation of the measure of membership of the element $x$ to operands 
$B_{1}, \ldots, B_{n}$ and to the result of the operation $(r) B_{i}$ :

$$
M_{\substack{n \\(r) B_{i} \\ i=1}}(x)=\left|M_{B_{1}}(x), . ., M_{B_{n}}(x)\right|^{r}, r=\overline{1, n}
$$

The introduced operation $(r)$ is called the $r$-composition of fuzzy sets $B_{1}, B_{2}, \ldots, B_{n}$. Thus, the measure of membership of the element of the $r$-composition of fuzzy sets is defined as an ordinal LD of rank $r$ from the set of measures of membership of this element to particular sets. In the particular case when $r=1$, we obtain the one-composition of fuzzy sets which coincides with their intersection. In another particular case when $r=n$, we obtain $n$-composition of fuzzy sets that coincides with their union. In general case, for $2 \leq r \leq n-1$ (which is the case if $n \geq 3$ ) the $r$-composition is a new operation that is essentially different from both the union and intersection of fuzzy sets. More precisely, this operation is intermediate between operations of union and intersection, which follows from the obvious inequalities

$$
M_{\substack{n-1 \\ n_{i}}}(x)=\bigwedge_{i=1}^{n} M_{B_{i}}(x) \leq M_{\substack{n \\(r) B_{i} \\ i-1}}(x)=\left|M_{B_{i}}(x)\right|^{r} \leq \bigvee_{i=1}^{n} M_{B_{i}}(x)=M_{\bigcup_{i=1}^{n} B_{i}}(x), r=\overline{1, n}
$$

It can be seen from (9) that the operation of the $r$-composition of fuzzy sets is stronger than the operation of their union but weaker than their intersection, i.e.,

$$
x \in \underset{i}{\cap} B_{i} \rightarrow \underset{i}{x} \underset{i}{(r)} B_{i} \rightarrow x \in \underset{i}{\cup} B_{i}
$$

As $r$ increases from 1 to $n$, the "strength" of the $r$-composition is almost reduced to the strength of the operation of the intersection of sets, and, while $r$ decreases from $n$ to 1 , this strength increases tending to the strength of the operation of interaction from set theory.

Properties of the composition of fuzzy sets and its relation with the union and intersection of fuzzy sets are a consequence of following considerations. Being a generalized operation of union and intersection of such sets, the $r$-composition of fuzzy sets can be represented in the form of their superposition. Indeed, writing the LD in the right-hand of (8) in detail, we obtain according to (5)

$$
M_{\substack{(r) B_{i} \\ i=1}}(x)=\bigvee_{i_{1} \neq \ldots \neq i_{n-r+1}}\left(M_{B_{i_{1}}}(x) \wedge \ldots \wedge M_{B_{i_{n-r+1}}}(x)\right)
$$

However, according to (2), the FL conjunction (disjunction) of measures of the membership of the element of fuzzy sets corresponds to the intersection (union) of these sets. Therefore, (9) implies the expression of the $r$-composition in the form of the union of intersections of sets

$$
(\stackrel{n}{r}) B_{i}=\bigcup_{i_{1} \neq \ldots \neq i_{n-r+1}}\left(B_{i_{1}} \cap \ldots \cap B_{i_{n-r+1}}\right) .
$$


Similarly, we obtain the dual expression of the $r$-composition in form of intersection of unions of sets

$$
\stackrel{n}{r} r) B_{i}=\bigcap_{i_{1} \neq \ldots \neq i_{r}}\left(B_{i_{1}} \cup \ldots \cup B_{i_{r}}\right)
$$

The $r$-composition of fuzzy sets must satisfy the following laws: the distributive law relative to the intersection and union

$$
\left.A \cap\left(\underset{i=1}{(\stackrel{r}{r}) B_{i}}\right)=\underset{i=1}{\stackrel{n}{r}}\right)\left(A \cap B_{i}\right), \quad A \cup\left(\underset{i=1}{(\stackrel{n}{r}) B_{i}}\right)=\underset{i=1}{(\stackrel{n}{r})}\left(A \cup B_{i}\right)
$$

law of complex (repeated) composition

$$
\left(\begin{array}{c}
n \\
\left(r_{1}\right) B_{i}
\end{array}\right)(p)\left(\underset{i=1}{\left(r_{2}^{n}\right) B_{i}}\right)(p) \ldots(p)\left(\underset{i=1}{\left.\left(\begin{array}{c}
n \\
r_{k}
\end{array}\right) B_{i}\right)}\right)=\left(r_{p}\right) B_{i}, r_{1}<r_{2}<\ldots<r_{k}, p \leq k
$$

and the generalized de Morgan law

$$
\overline{\left(\begin{array}{c}
n \\
r
\end{array}\right) B_{i}}=\left(n-r_{i=1}^{n}+1\right) \overline{B_{i}}
$$

To prove the first (second) law in (14), it is sufficient to express in it the composition of sets $(\stackrel{n}{r}) B_{i}$ in the form (12) (in the form (13)). Then, we have to apply the distributive law of union relative to $i=1$

the intersection (intersection relative to the union). To prove law (16), it is sufficient to express in it the composition of sets $\stackrel{n}{r} \underset{i=1}{r} B_{i}$ in form (12) or (13). Then, we have to write in detail the expression under the negation sign using de Morgan law. The validity of (15) follows from the definition of the $r$-composition of sets (8).

\section{Method for Solving the Problem}

According to Sec. 2, to solve the posed problem of integrating individual evaluations of the particular experts $i$ expressed in the form of fuzzy sets $B_{i}, i=\overline{1, n}$, into a cooperative evaluation in the form of a fuzzy set $B$, we construct and use the most representative expert of this collective. This expert executes the function of ordered choice from the measures of membership $M_{B_{i}}(x), i=\overline{1, n}$ for any given point $x$ to individual evaluations $B_{i}$; and the result of this choice is the measure of membership $M(x)$ of the point $x$ to the cooperative evaluation $B$.

To be precise, the most representative expert executes the function of choice of the $r$-th 
element in increasing order among the elements of the finite set $M(x)=\left\{M_{B_{1}}(x), M_{B_{2}}(x), \ldots, M_{B_{n}}(x)\right\}$ or, equally, finds the ordinal logical determinant of rank $r$

$$
|M(x)|^{r}=\left|M_{B_{1}}(x), M_{B_{2}}(x), \ldots, M_{B_{n}}(x)\right|^{r}
$$

Comparing (17) with (8), we come to the conclusion that if we use terms of fuzzy sets, rather than measures of membership, the most representative expert of a collective of experts performs an operation on fuzzy sets (individual expert evaluations) $B_{i}, i=\overline{1, n}$, in form of $r$-composition of these sets (7) with an appropriate value of $r$, and result of this operation is a fuzzy set (cooperative evaluation) $B$. The choice of an appropriate value of $r$ is obvious here, since (Sec. 1), at each point $x$, the most representative expert should choose the measure that is sufficiently distant from extreme evaluations and has average properties. This measure is chosen among the measures $M_{B_{i}}(x)$ of its membership to individual evaluations $B_{i}$ given by experts $i$ as measure of its membership to the cooperative evaluation $I$. This means that we have

$$
1<<r<n, \quad r \approx n / 2 .
$$

Thus, the integration of individual evaluations of experts $i$ in the form of fuzzy sets $M_{B_{i}}(x), i=\overline{1, n}$, into cooperative evaluation in the form of the fuzzy set $B$ is the operation of $r$-composition of pointed sets $B_{i}$ with an appropriate value of $r$ in accordance with (18). It follows from (17) that the measure of membership of an arbitrary element $x$ to the cooperative evaluation $B$ of the situation is calculated in our approach by an ordinal logical determinant of rank $r$ of the collection of the measures of membership of this element to the individual evaluations of experts $B_{1}, \ldots, B_{n}$. Therefore, our approach to the integration of individual evaluations into a cooperative one can be called the rank approach. Formula (12) of the decomposition of the cooperative evaluation $\underset{i=1}{(r)} B_{i}$ into the individual $B_{1}, \ldots, B_{n}$ ones implies that the rank approach is equivalent to the following procedure: 1) all possible representative subsets that consist of $n-r+1$ experts are selecting; 2) in each representative subset, its own cooperative evaluation is obtaining by the method of intersection of the individual evaluations [1]; 3) the best (with the maximal measure of membership $M_{B}(x)$ ) evaluation obtained in the representative subsets is taking as a final cooperative evaluation. If we use another formula for the decomposition of the cooperative evaluation into individual ones (13), then the rank approach is equivalent to the following procedure: 1) all possible "check sets" of experts that each consist of $r$ experts are selecting; 2) in each "check set", its own representative evaluation is obtaining by method of integration of individual evaluations [1]; 3) the intersection of all evaluations $B$ is taken as a cooperative evaluation. 


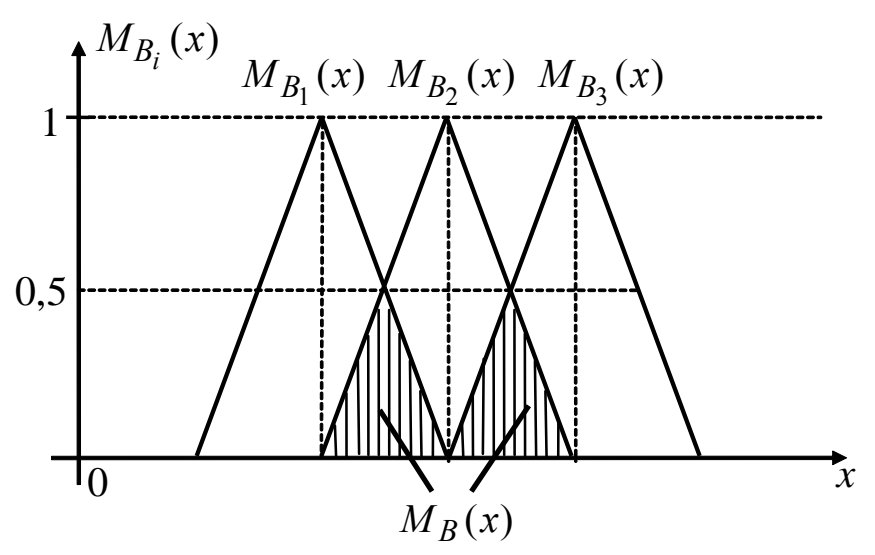

Fig. 1

Example. Three experts $i=\overline{1,3}$ give individual evaluations of the same situation in the form of fuzzy sets $B_{1}, B_{2}$ and $B_{3}$, whose membership functions $M_{B_{i}}(x), i=\overline{1,3}$ are shown in Fig. 1 . It is required to integrate the individual evaluations $B_{i}$ into a cooperative one $B$.

We will follow the rank approach. In our case, the number of experts $n=3$ and, according to (17), (18), the membership function $M_{B}(x)$ ofe cooperative evaluation $B$ realized by the most representative expert is

$$
M_{B}(x)=\left|M_{B_{1}}(x) M_{B_{2}}(x) M_{B_{3}}(x)\right|^{2}
$$

In other words, the cooperative evaluation $B$ is obtained from the individual evaluations $B_{1}, B_{2}$ and $B_{3}$ by the choice of the middle of three measures of membership given by individual evaluations at each point $x$ and the result is presented in the Fig. 1 by the dashed line.

Note that, if the method of intersection [1] is applied to our example, an empty set is obtained as cooperative evaluation $B$; i.e., there is no evaluation in this case.

\section{Conclusion}

The introduced operation of the $r$-composition of fuzzy sets is not a generalization of operations with conventional sets and does not turn into them when we pass from fuzzy sets to conventional ones. It is a new operation that has no analogies among usual set-theoretic operations. The operation of the $r$-composition shows that there is no gap between union and intersection of fuzzy sets. Both are $r$-compositions with different values of the index $r$. Because of the essential novelty of the operation of the $r$-composition, the opportunity to achieve more efficient logical inference and decision making arises.

\section{References}

1. Bellman, R.E., Zadeh, L.A., "Decision Making in Fuzzy Environment”, Management 
Science, 4, Vol. 17, 1970.

2. Dubois, D., Prade, H., Fuzzy Sets and Systems: Theory and Applications, New York: Academic Press, 1980.

3. Zimmermann, H.J., Fuzzy Sets, Decision Making and Expert Systems, Kluwer Academic, 1986.

4. Yager, R.R., "Connectives and Quantifiers in Fuzzy Sets”, Fuzzy Sets and Systems, Vol. 40, 1991.

5. Zadeh, L., The Concept of Linguistic Variable and Its Application to Approximate Reasoning, New York: Elsevier, 1973.

6. Levin, V.I., Structural-Logical Methods of Analysis of Complex Systems. Moscow: Nauka, 1987 [in Russian]. 